\section{POS0675 THE COMPARATIVE 3-YEAR RETENTION RATE OF TARGETED-SYNTHETIC AND BIOLOGIC DRUGS FOR RHEUMATOID ARTHRITIS: REAL-LIFE DATA FROM THE ITALIAN GISEA REGISTRY}

E. G. Favalli ${ }^{1}$, F. lannone ${ }^{2}$, E. Gremese ${ }^{3}$, R. Gorla ${ }^{4}$, R. Foti ${ }^{5}$, F. Conti ${ }^{6}$, M. Rossini ${ }^{7}$, E. Fusaro ${ }^{8}$, F. P. Cantatore ${ }^{9}$, C. Montecucco ${ }^{10}$, M. Sebastiani ${ }^{11}$, A. Cauli ${ }^{12}$, G. Ferraccioli ${ }^{13}$, G. Lapadula ${ }^{14}$, R. Caporali ${ }^{1,15}$ on behalf of Gruppo Italiano di Studio sulla Early Arthritis, GISEA. ${ }^{1}$ ASST Gaetano Pini-CTO Institute, Division of Clinical Rheumatology, Milan, Italy; ${ }^{2}$ University of Bari, Department of Medicine, Rheumatology Unit, Bari, Italy; ${ }^{3}$ Fondazione Policlinico Universitario A. Gemelli-IRCCS, Università Cattolica del Sacro Cuore, Rheumatology Division, Rome, Italy; ${ }^{4}$ Spedali Civili, UO Reumatologia e Immunologia Clinica, Brescia, Italy; ${ }^{5}$ Policlinico Vittorio Emanuele, Department of Medicine, Rheumatology Unit, Catania, Italy; ${ }^{6}$ Sapienza University of Rome, Dipartimento di Scienze Cliniche, Internistiche, Anestesiologiche e Cardiovascolari-Reumatologia, Rome, Italy; ${ }^{7}$ University of Verona, Department of Medicine, Rheumatology Unit, Verona, Italy; ${ }^{8}$ Città Della Salute E Della Scienza Hospital, Rheumatology Unit, Turin, Italy; ${ }^{9}$ University of Foggia Medical School, Department of Medical and Surgical Sciences, Rheumatology Clinic, Foggia, Italy; ${ }^{10}$ IRCCS Policlinico San Matteo Foundation, University of Pavia, Rheumatology Unit, Pavia, Italy; ${ }^{11}$ University of Modena and Reggio Emilia, Rheumatology Unit, Modena, Italy; ${ }^{12}$ Policlinico of the University of Cagliari, Department of Medical Sciences, Rheumatology Unit, Cagliari, Italy; ${ }^{13}$ Gruppo Italiano di Studio sulla Early Arthritis, GISEA, Rome, Italy; ${ }^{14}$ Gruppo Italiano di Studio sulla Early Arthritis, GISEA, Bari, Italy; ${ }^{15}$ Università degli Studi di Milano, Department of Clinical Sciences \& Community Health, Research Center for Adult and Pediatric Rheumatic Diseases, Milan, Italy

Background: Long-term observational data on the real-life use of JAK inhibitors (JAKis) for rheumatoid arthritis (RA) and their comparison with biological drugs are still very limited. Large population-based registries have been increasingly used to investigate the performance of targeted drugs in a real-life setting. Objectives: The aim of this study is to evaluate and compare the 3-year retention rate of JAKis, TNF inhibitors (TNFis) and biologic drugs with other mechanisms of action (OMAs) in the large cohort of RA patients included in the Italian national GISEA registry.

Methods: Data of all RA patients treated with targeted synthetic or biologic drugs were prospectively collected in the Italian multicentric GISEA registry. The analysis was limited to patients who started a first- or second-line targeted drug in the period after the first JAKi was marketed in Italy (1 $1^{\text {st }}$ December 2017). The 3-year retention rate was calculated by the Kaplan-Meier method and compared between different drug classes by a log-rank test. A descriptive analysis of reasons for discontinuation was performed.

Results: The study population included 1027 RA patients $(79.8 \%$ females, mean age $[ \pm S D] 56.9[ \pm 13.5]$ years, mean disease duration $9.8[ \pm 9]$ years, mean baseline SDAI 17.5 [ \pm 11.9$]$, ACPA positive $67.4 \%$, RF positive $62.7 \%$ ) who received JAKis (baricitinib or tofacitinib, $n=297)$, TNFis $(n=365)$, or OMAs $(n=365)$ as first or second targeted drug. Main baseline characteristics of study population were overall well balanced between treatment groups. Retention rate was numerically but not statistically higher $(p=0.18)$ in patients treated with JAKis compared with TNFis or OMAs $(80.6,78.9$ and $76.4 \%$ at 1 year and $73,56.8$ and $63.8 \%$ at 3 years, respectively) (Figure 1). Drug survival was significantly higher in patients receiving concomitant methotrexate (MTX) compared with monotherapy only in TNFis (66.8 vs $47.1 \%, \mathrm{p}=0.04$ ) but not in JAKis ( 76.1 vs $70.1 \%, \mathrm{p}=0.54$ ) and OMAs (66.1 vs $61.9 \%, p=0.41$ ) group. Therapy was discontinued in a total of 211 patients because of ineffectiveness $(n=107)$, adverse events $(n=88)$, or compliance/other reasons $(n=16)$. The most frequent reason for treatment withdrawal was ineffectiveness in both JAKis ( $n=30$ out of 56 ) and TNFis ( $n=45$ out of 74 ) groups, whereas OMAs were discontinued more frequently because of adverse events ( $n=41$ out of 81 ).

Conclusion: Our data confirmed in a real-life setting a favorable 3-year retention rate of all available targeted mechanisms of action for RA therapy. As expected,

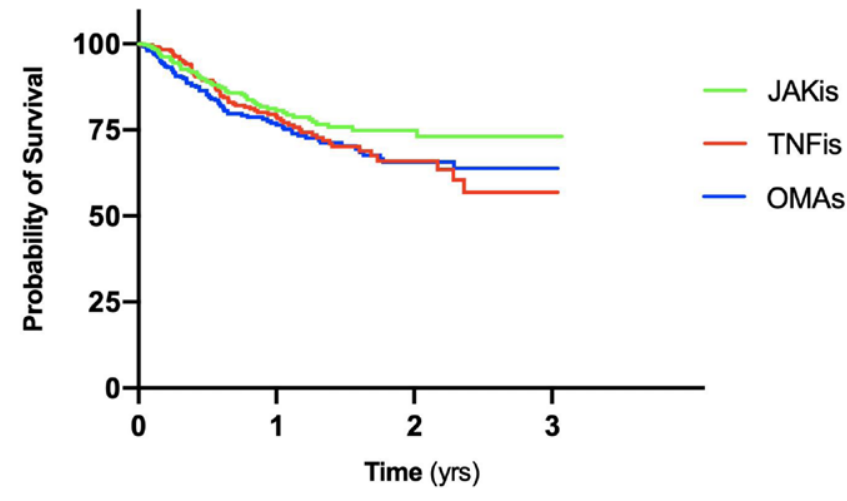

Figure 1. Three-year retention rate by treatment group concomitant MTX significantly impacted persistence on therapy of TNFis only. Discontinuations of JAKis for adverse events were infrequent overall, confirming the safety profile observed in randomized clinical trials.

Disclosure of Interests: None declared

DOI: 10.1136/annrheumdis-2021-eular.3557

\section{POS0676 SURVIVAL OF THE FIRST COURSE OF BIOLOGIC OR JAK INHIBITOR IN RHEUMATOID ARTHRITIS: ASSOCIATION WITH THE CHOICE OF AGENT AND CONCOMITANT CONVENTIONAL SYNTHETIC DMARDS}

M. Bredemeier ${ }^{1,2}$, A. Duarte ${ }^{1}$, M. Pinheiro ${ }^{1}$, B. Stadler ${ }^{2}$, J. C. Macieira ${ }^{1}$, R. Ranza ${ }^{1}$, J. Miranda ${ }^{1}$, V. Valim², G. Castro ${ }^{1}$, M. Bertolo ${ }^{2}$, M. D. F. Sauma ${ }^{2}$, V. Fernandes ${ }^{2}$, A. Medeiros ${ }^{2}$, R. Botelho ${ }^{2}$, C. Brenol ${ }^{2}$, D. Negrão Gonçalo Dias $^{2}$, H. Carvalho ${ }^{2}$, S. Studart ${ }^{2}$, G. Da Rocha Castelar Pinheiro ${ }^{2}$, L. Rocha ${ }^{2}$, I. Pereira ${ }^{2}$, M. Ohira Gazzeta ${ }^{2}$, A. Maria Kakehasi ${ }^{2}$, P. Louzada Jr², A. L. S. Hayata ${ }^{2}$, F. Pina ${ }^{2}$, C. Lupo ${ }^{2}$, L. Balarini², I. Silveira², S. Kowalski² ${ }^{2}$ D. Titton ${ }^{2}$ R. Chakr², A. Ranzolin², I. Laurindo ${ }^{1}$, R. Xavier ${ }^{1}$ on behalf of BiobadaBrazil. ${ }^{1}$ BiobadaBrasil, Brazilian Society of Rheumatology, SAO PAULO, Brazil; ${ }^{2}$ BiobadaBrasil, Brazilian Society of Rheumatology, Sao Paulo, Brazil

Background: After failure of conventional synthetic disease modifying anti-rheumatic drugs (csDMARDs) in the therapy of rheumatoid arthritis (RA), treatment may be escalated to biologic (bDMARDs) or JAK inhibitors (JAKi) (1). Analysis of drug survival can provide useful information on the effectiveness of these therapeutic schemes.

Objectives: to evaluate the association of the choice of therapeutic agent with the survival of treatment course in RA patients receiving their first bDMARD or JAKi. Methods: BiobadaBrasil is a multicentric registry-based cohort study of Brazilian patients starting their first bDMARD/JAKi (2). This analysis includes RA patients recruited from Jan 2009 to Oct 2019, followed-up over the first course of treatment with a bDMARD/JAKi until censoring (latest date, Nov 19, 2019) or occurrence of the outcome of interest. A treatment course is defined as a period during which the medication scheme does not change, except for dose adjustments. The primary outcome was the interruption of treatment course for any reason (except for pregnancy or disease remission); interruption of treatment due to adverse events (AEs) or death and due to inefficacy served as secondary outcomes. Multivariate Cox proportional hazards models were used for analyses. Results: In total, 1177 patients (3800 patient-years [PY]) were enrolled. The overall incidence of treatment interruption was 17.5/100 PY. Adalimumab was the most frequently prescribed agent, followed by infliximab $(n=267)$. The hazards ratios (HR) of the primary and secondary outcomes are presented in Table 1. Figure 1 compares the survival of treatment curves of different bDMARDs/JAKi.

Table 1. Hazard ratios (HR) of interruption of therapy course of each therapeutic agent (the reference category for bDMARDs/ JAKi is infliximab). Results are HR, 95\% Cls, and P values*.

Agent (number of Interruption for any Interruption due to adverse patients) reason (665 events) events or death (196 events) Interruption due to inefficacy (319 events)

Adalimumab ( 354$) 0.83$ ( 0.68 to 1.01 ), $P=0.68$ ( 0.48 to 0.96$), P=0.029$ 0.062

$1.08(0.80$ to 1.44), $P=0.621$

Etanercept (257) 0.81 (0.66 to 1.01), $\quad 0.56$ (0.37 to 0.83 ), $P=0.004 \quad 0.93$ (0.68 to $\mathrm{P}=0.063$

$0.93(0.68$ to
$1.29), P=0.674$ 1.32 ( 0.74 to

2.35), $\mathrm{P}=0.350$ $P=0.185$

$.07(0.53$ to

2.15), $P=0.849$

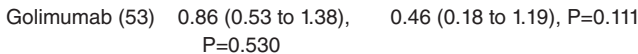
.54 (0.30 to

$0.89(0.41$ to

1.96), $P=0.779$

(59) $\quad P=0.047$

Rituximab (48) $\quad 0.87$ (0.55 to 1.37 ), $\quad 0.48$ (0.20 to 1.18$), P=0.109$ $\mathrm{P}=0.540$

Abatacept (30) $\quad 0.52$ (0.25 to 1.07$), \quad 0.46(0.14$ to 1.56$), P=0.215$ $\mathrm{P}=0.077$

$0.58(0.26$ to

1.34), $P=0.205$

$0.46(0.14$ to 1.52), $P=0.203$

Tocilizumab (29) $0.29(0.14$ to 0.63$), \quad 0.40(0.12$ to 1.30$), P=0.126 \quad 0.28(0.09$ to $\mathrm{P}=0.002$

Methotrexate $\quad 0.95$ (0.79 to 1.14$), \quad 0.86(0.62$ to 1.19$), P=0.362$

(792) $\quad \mathrm{P}=0.561$

Leflunomide (497) 1.17 (0.99 to 1.39), 1.44 (1.06 to 1.96 ), $P=0.020$ $P=0.061$

Sulfasalazine (48) 1.18 (0.80 to 1.75$)$, $P=0.401$

Antimalarials $\quad 0.80(0.65$ to 0.98$)$, $\mathrm{P}=0.027$

$0.90), P=0.033$

$0.98(0.75$ to 1.28), $P=0.860$ $1.02(0.80$ to 1.30), $P=0.856$ 0.85 (0.45 to 1.59), $P=0.605$ 0.67 ( 0.50 to 0.92), $P=0.011$

* All tests adjusted for other variables presented in the table and for age, baseline DAS28, disease duration, gender, smoking, seropositivity (RF/anti-CCP), previous malignancy, diabetes, hypertension, hypercholesterolemia, renal failure, ischemic cardiomyopathy, COPD, heart failure, use of corticosteroids, starting year, hypercholesterolemia, osteoporosis, hepatitis B and $\mathrm{C}$. 